\title{
'Community navigators': making a difference by promoting health in culturally and linguistically diverse (CALD) communities in Logan, Queensland
}

\author{
Saras Henderson $^{\mathrm{A}, \mathrm{C}}$ and Elizabeth Kendall ${ }^{\mathrm{B}}$ \\ APopulation and Social Health Research Program, Griffith Health Institute, School of Nursing and Midwifery,

\begin{abstract}
A key component of the 2011 Australian National Health Reform, via the Access and Equity Policy, is to improve access to quality health services for all Australians including CALD communities. Awareness has been raised that certain CALD communities in Australia experience limited access to health care and services, resulting in poor health outcomes. To address this issue, the Community Navigator Model was developed and implemented in four CALD communities in Logan, Queensland, through a partnership between government and non-government organisations. The model draws on local natural leaders selected by community members who then act as a conduit between the community and health service providers. Nine 'navigators' were selected from communities with low service access including the Sudanese, Burmese, Afghan and Pacific Islander communities. The navigators were trained and employed at one of two local nongovernment organisations. The navigators' role included assessing client needs, facilitating health promotion, supporting community members to access health services, supporting general practitioners (GPs) to use interpreters and making referrals to health services. This paper explores the 'lived experience' of the navigators using a phenomenological approach. The findings revealed three common themes, namely: (1) commitment to an altruistic attitude of servility allowing limitless community access to their services; (2) becoming knowledge brokers, with a focus on the social determinants of health; and (3) 'walking the walk' to build capacity and achieving health outcomes for the community. These themes revealed the extent to which the role ofCALD community navigators has the potential to make a difference to health equity in these communities, thus contributing to the Australian National Health Reform.
\end{abstract}

Additional keywords: health service access and equity, national health reform, phenomenology, role of community navigators.

Received 3 May 2011, accepted 11 August 2011, published online dd mmm yyyy

\section{Introduction}

Across the world, there are vast disparities in health service delivery and health outcomes for culturally and linguistically diverse (CALD) communities. In Australia, for example, members of communities from countries such as Sudan, Burma (Myamar), Afghanistan and the Pacific Islands experience less access to health care and poorer health outcomes compared with the Australian-born population and immigrants from Englishspeaking countries (Federation of Ethnic Communities Councils of Australia 2009). The report of the Federation of Ethnic Communities Councils of Australia (Federation of Ethnic Communities Councils of Australia 2009) stated that the difficulties associated with navigating our health system render it incapable of meeting the needs of CALD consumers, effectively preventing them from taking an active role in their own health care. As a result of access difficulties, members of some CALD communities may only seek health care and support when their health condition has reached a crisis point, a situation that leads to less than optimal health outcomes (Federation of Ethnic Communities Councils of Australia 2009). This situation detracts from their overall well-being and increases their vulnerability to disease.

A systematic literature review (Henderson et al. 2011) indicated that one successful approach to reducing CALD health disparities is the use of community-based bilingual health workers. These health workers are natural leaders, selected by their communities, because they are in a position to promote positive approaches to health and minimise disease risks within their communities. Most importantly, they are able to assist their people to navigate the complex Australian health system, 
enabling them to access appropriate and timely services. Eng and Parker (2002) claimed that lay health workers are becoming increasingly recognised as critical tools for reducing people's vulnerability to disease and illness. Partnerships between formal health services and community-based social networks can facilitate appropriate care that is specific to the particular needs of each community (Eng and Parker 2002). Eng and Parker further argued that lay models of health promotion do not seek to replace the more specialised role of health professionals, but aim to provide additional community-based care and social support to CALD community members.

In terms of health outcomes, a recent systematic review (Lewin et al. 2007) identified sufficient evidence to conclude that using this lay 'community health worker' approach may promote greater uptake of prevention strategies, such as immunisation, screening and health monitoring. Mixed findings were apparent in relation to the ability of the model to promote culturally appropriate services, but as this was not the direct intention of most studies, it was often measured as an afterthought. There was sufficient evidence that intense one-to-one advocacy or support from a culturally appropriate community member may be the most effective style of intervention to improve access for CALD communities. Moreover, lay health worker programs have been considered to be effective in precipitating health behaviour change (Altpeter et al. 1999).

Based on available literature, and in partnership with government and non-government agencies, we developed a lay cultural health worker model for use in Logan, Queensland. Logan was selected because the 2006 census indicated that this region hosted a sizable number (25.3\%) of non-English-speaking immigrants and refugees especially from African, Middle Eastern and Asian countries. There was also a large Pacific Islander community (consisting of Tongan, Samoan, Maori and Cook Island people) living in Logan (Australian Bureau of Statistics 2001). In our project, the lay health workers became known as community navigators due to their primary role as a culturally appropriate conduit between the community and the health system. However, they also facilitated local health promotion and disease prevention activities and supported communities to become more engaged in local health service planning. In terms of facilitating access to health services, the navigators assessed clients' needs, conducted health education sessions, supported community members to attend general practitioners (GPs) and health services, educated communities about Australian health systems, supported GPs to use interpreters and made referrals to appropriate health services when needed. In line with existing research, it was anticipated that the navigators would form a sustainable bridge between CALD communities and health service providers and build capacity to facilitate community empowerment (Altpeter et al. 1999; Bishop et al. 2002; Yu et al. 2007).

Nine bilingual community navigators were selected by their communities: two (male and female) from the Sudanese community, two (both female) from the Burmese community, two (male and female) from the Afghan community, and three (two female and one male) from the Pacific Islander community. The navigators were trained and employed by the partner nongovernment organisations. The training for the navigators consisted of five 3-h modules (Kelly et al. 2010) conducted through the university followed by a one-year part-time graduate certificate in community development and ongoing weekly learning circles designed to support their practice (Table 1).

This paper presents the findings of an exploration into the 'lived experience' of the navigators during the first 18 months of their operation in Logan, Queensland. Although there is significant evidence to support the potential value of the lay cultural health worker model(Henderson et al. 2010), evaluations of such programs rarely describe the nature of the work that constitutes a lay health worker's role or the experience of those workers as they undertake the role (Altpeter et al. 1999). In examining community-based responses to health disparities, it is important to understand how these lay initiatives function. Specifically, by gaining information about the exact nature of the community navigator role, health service providers will be able to better understand the complex and ever changing nature of the role and the requirements or circumstances that contribute to its enactment. A close examination will facilitate identification of common elements of the role that can be formalised and sustained in future. The current study aimed to identify these common elements by using phenomenology to build a picture of the actual work of community navigators in action.

\section{Methods}

The phenomenological approach was used to explore the 'lived experience' of the community navigators. According to Reid et al. (2005), phenomenology is useful in offering researchers insights into how people make sense of the world in a specific context. Phenomenology as a method is particularly effective in eliciting people's perceptions and interpretations of situations and events from their own perspective away from normal assumptions of everyday life (Smith 2007). As the epistemology of phenomenology is one of personal knowledge and subjectivity, it was appropriate for the current study as we needed to explore and extract the essence of how the navigators perceived their lived experience of the role with respect to their actions and motivations.

\section{Data collection and analysis}

Following ethics approval from Griffith University Ethics Committee (HSV02/07HREC) and participant consent, in-depth

Table 1. Community navigator training modules

Module one Introduction to the community navigator model of support and culturally diverse models of health

Module two Strategies for supporting community members and building community capacity; health service systems and health promotion

Module three Prioritising and goal setting; communication skills development; advocacy; the social determinants of health, such as housing, income, unemployment

Module four Working alongside health service planners, funders and providers to improve the provision of culturally safe services

Module five Strengthening and formalising community partnerships to ensure coordinated and complementary services and activities to the target CALD communities 
interviews were conducted with the nine navigators. The questions were semi-structured and open-ended to encourage unrestricted responses. Participants were asked questions such as - Can you describe your role? Can you describe a typical day as a navigator? Can you describe any challenges? How do you feel about these? Can you share with me how you promote health? Can you describe how the role is affecting you and the community? What are your views about the role and its future? The taped interviews were transcribed and analysed using Giorgi's (1985) four steps of analysis (Table 2).

The interpretation of the data was checked with the participants to ensure reported findings represented their true experiences (Streubert-Speziali and Carpenter 2006). An audit trail was created to ensure that the thought processes and evidence that led to the conclusions made by the researchers could be confirmed and replicated. Specifically, the researchers kept a journal of their thoughts and ideas as they progressed, allowing them to bracket their prior beliefs and assumptions, ensuring that they did not shape the analysis of the data (Crotty 1996).

\section{Results}

Three common themes emerged that described the lived experience of the community navigators. These themes included: (1) commitment to an altruistic attitude of servility allowing limitless community access to their services; (2) becoming a knowledge broker with a focus on the social determinants of health; and (3) 'walking the walk' to build capacity and achieve health outcomes for the community. These three themes dominated the lived experience of the nine navigators as they translated their role into practice. Although each navigator presented unique narratives that illustrated these common themes, they adequately captured most of their experiences. Member checks with participants also confirmed the validity of these themes.

Theme 1: Commitment to an altruistic attitude of servility allowing limitless community access to the navigators

Participants were employed for only $11 \mathrm{~h}$ per week, but the nature of their work meant that all participants faced the challenge of having to work longer hours, some averaging $40-50 \mathrm{~h}$ per week.

Table 2. Giorgi's (1985) four steps of analysis

Step one The researchers dwelt on and read the descriptions in the transcripts to get a sense of the whole story as presented by each participant

Step two Once the sense of the whole was grasped, the researchers highlighted those statements that focussed on the participants 'lived experience' of being a community navigator. These were labelled 'meaning units'

Step three After the 'meaning units' were illuminated, the researchers inductively clustered and collapsed similar 'meaning units' into conceptual categories that captured the subjective reflections as described by the participants

Step four In the final step all the conceptual categories were collapsed and synthesised into themes that depicted the essence of the shared insights about participants' lived experience of the role of community navigator
They described how their community members had no-one else to assist them, so the navigators felt 'pressured' to help outside of their designated hours of work. Participants believed that they were chosen by their people to be navigators, so needed to be available to serve their communities regardless of the number of hours it took to fulfil their role. Participants voluntarily allowed community members to have unlimited access to their services (day, night or weekends) often to the detriment of time with their own family. They believed they were not renumerated adequately for the number of hours they worked, but the number of hours they worked prevented them from accepting other employment to supplement their incomes. Complicating this dilemma was the fact that they could not limit the duration of their work to suit the number of hours for which they were paid. Their altruistic behaviour was described in this way:

They [community members] call me all the time-day and night wanting my help... when I was with my family at night they call me. When I tell them I' $m$ now with my wife, I' $m$ with my kid you can't call me at this time of night. They complain, why, why? You don't want us to call you. I say, okay because you locate me then I do it at this time. I know they [community members] have nowhere to go. I have to serve them like that ...

I won't be far off the mark if I say I work 40 to 60 hours a week and weekends. They [community members] rang me on Sunday morning and said - ' can you come with us to the hospital? We' re going in to see the young one [child]'. I said, 'What do you need?' and they said 'We want to talk with a nurse and we feel that there's a lot of things that we want to ask but we don' $t$ know how to ask questions that will relate to the condition or understand what a doctor is saying'. This happens all the time and I have to help them because there is no one else!

Although participants voluntarily adopted this servile attitude so they could be available to the community on a $24 \mathrm{~h}$ basis, their statements reflected a sense of concern about not being able to sustain such accessibility over time.

The sad thing is they [employing agency] want me to be the link for the community but they pay us minimum hours. They want my full commitment to make sure this service [community navigator] works. That's fair enough but they payme peanuts. It takes hours to support one person. I have a family to feed. I' d love to be there for my people but at the end of the day, it doesn't pay my mortgage. I can't pick up other work, there is no time...

As it was built through a short-term demonstration project, the role of community navigator was not officially recognised or adopted by government or local authorities, leaving participants worried that they would have to continue their work with little or no government funding in future. Although they were developing sustainability strategies (e.g. mentoring new navigators, spreading their skills, capacity building), they believed that, in these unfunded circumstances, their voluntary commitment to their communities would lead to burn out. This, in their view, would be detrimental for the community and would damage trust in the model. 
If the government knows there is a group [of community navigators] doing voluntary work helping the community, they will not give any funding, why should they if we are already doing the work for them in trying to keep the community healthy ... all the navigators have said regardless of whether or not they get paid they will continue with their work because people are aware of them now and they feel obliged to help their people... we are going to get tired and not be able to help much.

This theme has shown that community navigators altruistically volunteered their time to assist community members to access health and other services. They were aware that facilitating access for their communities would require considerable voluntary effort by community leaders, but this was not considered to be sustainable without sufficient government funding.

Theme 2: Becoming a knowledge broker for the social determinants of health

Participants reflected that since becoming navigators their main role had been to act as a knowledge broker for their community. Participants explained that they were constantly approached by the community for information about a range of situations that impacted on health. As a result, they experienced an overwhelming expectation to provide information and knowledge not only about health issues, but also about the broader social determinants of health, such as housing, employment and education. Participants supported this broad focus as they believed that these social determinants were critical to the health status of their communities and had to be dealt with before addressing more superficial factors, such as risk behaviours. They described situations where stress, imposed by the lack of housing, employment or educational opportunities, had directly contributed to poor health outcomes for their communities. They described how these broad factors also prevented the timely use of health services and hindered lifestyle change.

They [community members] come and complain about having headache, neck pain, shoulder pain, cough and cry all the time. When you refer them to doctor, doctor say it all comes from stress. Whether financial problem, lack of job and housing or Centrelink pushing them to work and they can' tbecause of lack of English. They cannot talk Englishstress. They cannot find a job - stress. They haven't got money - stress. Housing - the owner put the rent up stress!

Participants explained how they had extended their health promoting role beyond a focus on disease treatment to incorporate activities that addressed these social determinants of health. They identified the need to support community members in all facets of their lives rather than just concentrating on health. The importance of these broad social issues to the health of the communities could not be understated, according to the navigators.

Even though our role is health focussed, if we don' tsupport the community in bigger issues, the social determinants of health, then when we start having conversations around specific health issues such as healthy eating, healthy actions, being physically active, the community won't be receptive to that because in their [community] mind we only want to know them for our issues.

To gain the trust of the communities, most navigators spent a great deal of time sorting out issues pertaining to the social determinants of health. This activity was seen as an integral precursor to fulfilling their role as health navigators. Indeed, they were adamant that failing to address these broader issues would alienate them from their communities and limit their opportunity to address more specific health concerns.

For housing problems, I have to accompany them to the Housing Department to sort that out. I have to provide information about the policies of the Housing Department to this person, because sometimes they cannot read or understand. If somebody has been told that you wait, your name is on the waiting list and they say why? When I explain the policy and give them this information, they are okay.

People with diabetics, abdominal pains ... these are the people that we deal with and they have problems like housing, nojob, immigration, legal and I have to help them. The other day I had to go to immigration with them [community member] and help with the visa because they cannot speak the language and nobody help them. These people think we know everything and want our help.

It was evident that participants were recognised by their communities as knowledge brokers. Community members expected them to have answers to all their queries, regardless of the topic, a situation that challenged the navigators. Participants believed they had to continually acquire new knowledge in a broad range of areas, for example: the process of allocating community housing; Centrelink and immigration rules; job searching techniques; funding eligibility; and policies and procedures across several government departments. This task of broad knowledge acquisition required the allocation of extra time beyond their normal paid employment.

The other day this client was upset because she has been waiting for a flat [accommodation] and said to me the government has not given it to her, maybe nobodyliked her. So I had to ask the housing people and Centrelink about the policy so I can learn and give my client the right answer, this was not easy. We have to learn as well and that can be challenging!

Another challenge for participants was that they were expected to engage in a diverse range of culturally appropriate behaviours to meet the needs of sub-groups within their own communities. The level of error likely to be tolerated from the navigators appeared to be lower than would be tolerated from non-cultural workers. Participants explained that within their own cultures, there were different gender-based norms and beliefs, religious groups and language clusters. Despite having a cultural background, participants were not always cognisant of the specific requirements of sub-groups within their community. 
Participants, therefore, felt compelled to learn about a range of different cultural behaviours

Some challenges are due to cultural difference because we have different cultures. So I have to learn the culture of that group. If not there will be misunderstanding. For example, there are some who have their beliefs - we have a Muslim group and Christian group. Muslims have their beliefs and the Christians have our beliefs. If I want to talk to them I will have to learn about their culture ... even the greeting handshake can be a challenge ... this is hard.

As knowledge brokers, the navigators frequently referred community members to appropriate health and other services to improve health outcomes. Referral to GPs was considered to be crucial for timely medical treatment. This task required navigators to develop understanding of the way in which GPs operated and how community members who did not speak English could maximise the value of their time with the GPs.

Our role is to support our community by connecting them to various service providers. Can be a GP visit, can be Centrelink, can be a dietician, dentist, mental health, disability services and aged care services; however, our main referral point is the GP... we are the GP's helper and hopefully hospital visits will be lower for our people because they are now seeing the doctor sooner with our help.

Navigators stated they sometimes faced a challenge when referring community members to GPs because doctors often perceived them to be interpreters and expected them to take this role. The navigators felt uncomfortable about taking this role as they were not qualified to do so, but declining to interpret would mean that the medical visit was pointless. Other options were not available.

Some GPs they don't provide interpreters and we are expected to interpret. I say no, I am not an interpreter. You have to access the interpreting service. That's one thing I have been strong on because of legal reasons. I don't feel comfortable about saying this to the doctor and I feel frustrated because my client [community member] will have to wait for treatment.

Participants stated they were gradually making GPs aware of the need for interpreters to be readily available. They distributed business cards for accredited interpreters and noted that the doctors had started engaging interpreters. As a result, many nonEnglish speaking community members were now able to access medical consultation and treatment in a timely manner.

They [GP] call me. They say'I have this patient with me and I want to talk to him and he does not understand me. Can you tell me if there's anyone that can come and interpret?' I will say, okay. Then I have to provide a contact of the right person that can go and help.

Despite the stated role that was outlined for the community navigators and their training, this theme revealed that their primary task involved being knowledge brokers in areas that extended beyond the immediate health sector. According to the navigators, health and access to health services depended on the ability of community members to negotiate non-health systems. These systems were particularly important when they enabled community members to address their health concerns more adequately. Navigators quickly realised that the health messages they hoped to portray to the community were meaningless if broader social issues were not addressed.

\section{Theme 3: 'Walking the walk' to build capacity} and achieve health outcomes for the community

Participants described their role as one of 'walking the walk' or putting themselves in the shoes of the community members to help build capacity for the future. Participants perceived themselves as working with the community to facilitate empowerment, both for individuals and the entire community. Participants were able to apply their tacit knowledge about their people's local lifestyles to engage with them in a meaningful manner that enabled sensitive health-related topics to be raised easily. Participants explained that community members had confidence in them because they had been selected by the community. They also had first-hand experience of living in the community and were often managing the same issues faced by others.

I walk the walk with my community ... I know how hard it is for these people as they have limited understanding about health and other issues. I appreciate the fact that they know I have been there, done that and this gives them confidence to ask me for help. I think this is a big step if people understand that you know what it is like to face all the problems... you sort of get your foot in the door and you have the chance to work with them to bring about change for better health.

Participants described many perceived positive outcomes for community members as a consequence of them walking the walk with their communities. For example, in one community, mental illness was considered to be a curse and as a result community members did not seek medical help. The navigators understood this view, but were able to challenge it in a gentle and respectful way. Following information provided by the navigators, community members began to seek appropriate medical help for their family members with mental illness. The navigators then supported these families to access treatment, but noted that they were unable to do so until they had the full trust of the community.

Our community perceive mental illness as a curse, I'm slowly changing this through my role as community navigator and also I connect with my community and educate them. I convince them mental illness is like any other disease that needs to be treated. Because of my involvement this family who secretly kept their mentally ill teenager at home finally took him to the hospital for treatment and I think this was good.

Participants also highlighted the high incidence of so-called 'lifestyle diseases', such as diabetes, obesity, high blood pressure and cardiovascular disease in their communities. These same diseases had been experienced by navigators and their families, so it was easier for them to understand the challenges community 
members faced. Participants facilitated health promotion sessions with their communities about these diseases. However, rather than simply educating the community members, they worked alongside community members, learning and teaching simultaneously.

We've been doing breakfast sessions to promote healthy eating. So rather than having eggs and bacon for breakfast, we have pawpaw. We did that and they go this is good because this is how they used to eat back in the islands. We showed them [community] how to cut down on chicken and meat; but we know that as soon as they get home from that event, they'll get stuck into their takeaway [fast food]! They are aware of the need to be preventative for healthy living; even if they don't do it and it's a good start.

Participants believed that their approach would build community capacity in the long-term. They described how more of their people were becoming aware of health services and using these services more readily when needed. They reported that their communities were becoming empowered to manage their own health and, as a result, were beginning to engage in healthy behaviours, which would result in greater health equity in the long term. They provided examples of how their strategy of "walking the walk' had been so successful that they had now become a first point of contact for community members.

Services like this, you have gained that trust because to start with, it was new to the community but now, it's turned into something that people go to, they would pick up the phone and ring or even drop in to have a chat. We have made a difference. We made those connections within the community and to other services, so we are helping the community. People have got a positive frame of mind or at least improved their self-esteem. They have become different people than they were before. To be there for my people empowers me to appreciate my role as a navigator. I hope the navigator program continues.

This theme captured the crucial role community navigators can play in promoting access and equity in marginalised CALD communities simply by virtue of their strong and close linkages to the community. Interventions designed to address access and health disparities must be based on this common foundation of shared understanding and engagement to appropriately empower the recipients of any reform.

\section{Discussion}

In this paper, we identified three common themes that describe the lived experiences of nine community navigators working in a disadvantaged, outlying region of Queensland. This region is home to a large CALD population, which had for many years struggled with the difficulty of accessing appropriate and timely health services. The navigators reported a range of positive health outcomes for their communities as a result of their role. In particular, they described how they had raised awareness about health and built capacity for communities to manage their own health more effectively. Eng and Parker (2002) similarly found that through their extensive networks with community residents, agencies and the wider society, lay helpers were effective in improving local health behaviours, coordinating agencies and empowering their communities through capacity building. In the UK, Partridge et al. (2007) demonstrated that trained lay people were able to effectively deliver health education in their community. More importantly, they concluded that using lay people to promote health in the community can be a useful resource, especially when there is a shortage of health professionals, as is currently the case in Australia (Department of Health and Ageing 2011).

Three main strategies appeared to underpin the success of the navigators, namely, their willingness to adopt an altruistic commitment to serve their communities, their ability to gather and share knowledge about a wide range of topics including the broad social determinants of health, and their capacity to work in close connection with the community to develop trust and engage in local capacity building. By applying these strategies, the navigators were able to achieve significant success in making the community aware of healthy lifestyles and the need to seek medical assistance in a timely manner. They assisted community members to communicate with the doctors, and encouraged doctors to use accredited interpreters.

Most importantly, the navigators appeared to undertake a natural shift throughout the project from a focus on clinical treatment of diseases and modification of risk behaviour to a focus on addressing the broader social, environmental and systemic factors that impacted on people's ability to manage their own health. The navigators described how they were now working towards enabling their communities to become more involved in local health planning activities by informing them about the health system and building their capacity to engage with key decision makers. Over time, they expanded their role from simply supporting people with diseases to advocating for change in the broader health system and building capacity in their communities to engage in policy making.

The community navigator model concurs with the Australian National Health Reform 2011 (Department of Health and Ageing 2011), which aims to improve primary health care by focusing on the specific needs of local communities. Clearly, the community navigators knew how to meet the needs of their own communities. To do this, they adopted several strategies that empowered their communities. Specifically, they adopted an attitude of servility that enabled them to 'work for' their communities at all times. They acted as knowledge brokers, attending to broad factors that impacted on the health of their communities and they walked with their communities, building on their own understanding of the experiences and circumstances. These strategies were employed in addition to the more structured role of the navigators, which was to assist individuals to access health services.

Eng and Parker (2002) suggested that the main role of community-based health workers was to form a conduit between the community and health service providers. This was clearly the case for the navigators in this study. They connected community members with various health and social services, such as GPs, mental health services, disability services, and housing and welfare organisations. The navigators engaged with community members on a one-to-one basis to gain trust and reported that, as a result, they were successful in promoting healthy eating and increasing the likelihood of seeking medical advice in a timely 
manner. This finding concurs with the systematic review by Lewin et al. (2007), which reported that intense individual interaction with lay helpers was effective in promoting health. A good example provided by the navigators was in the area of mental health, where, as a consequence of navigators individually educating people about the need to seek medical help for mental illness, community members had begun to seek help more readily. Without the navigators, community members may not have been exposed to relevant services. Hibbert et al. (2008), in their review of grey literature on accessing mental health services by the community, reported that using a community-based approach with minority ethnic groups resulted in greater likelihood of such groups seeking help for mental health problems.

Participants in this study described their work as being primarily knowledge brokers, linking community members to information about the various systems that impacted on their lives. They also provided support and strategies to negotiate those systems. To perform this role, they were forced to adopt a broader approach to health than originally intended, recognising the significant impact of social determinants such as housing, education and employment. The need to extend the role of lay helpers to include a focus on the social determinants of health was also suggested by Bishop et al. (2002). Extending the scope of practice for the navigators temporarily increased their workload, but provided a longer-term opportunity to build capacity. Navigators all highlighted the importance of dealing with the broad context within which health occurred rather than focusing on disease only. They and their communities displayed a thirst for knowledge about how to build capacity at a broader level than that of individual self-management and a need to know how to influence the systems that affected their health.

Their main technique for performing their role was to "walk the walk' with their communities. At every opportunity, they emphasised the fact that they had experienced similar circumstances and understood the issues faced by community members. The development of trust was critical to their success. However, the process of gaining this trust entailed significant investment in learning about local circumstances and sub-cultures within their community as well as about the broad systems that affected them. They made the effort to engage fully with all members of their communities, but the implication of this effort was that they were available $24 \mathrm{~h}$ per day. The navigators voluntarily made themselves available to the community at all times because they knew there was no-one else available to help them. Simultaneously, the navigators had to gain the trust of the health professionals to convince them to use culturally appropriate methods. This need to walk within and between two distinct cultures was difficult. However, the navigators worried most about the sustainability of the role beyond the project, and the fact that they would feel pressured to continue even when they were no longer employed.

The three main strategies that underpinned the work of the navigators, although essential to their success, placed significant demands on them, necessitating long hours and extraordinary commitment. This extra workload impacted on their family time, causing them concern and fatigue. Clearly, the navigators were experiencing role overload and stress, despite the pleasure they gained from being able to support their communities. In her study about lay helpers, Gammonley (2009) also identified role overload as a common problem that impacted on the physical and mental health of lay workers. This study revealed a dilemma in that simply educating the navigators about how to place boundaries around their work actually placed them under greater pressure because doing so meant that they could not meet the expectation of their communities, and themselves. Ironically, the close connection they maintained with their community was the main source of this expectation, but was also their greatest success factor. Thus, if we wish to draw on lay helpers to address inequity and access barriers for CALD communities, we need to understand that the success of the role comes with serious personal implications for which they must be adequately compensated. It is important to address this dilemma, because the role offers such significant potential for addressing access barriers and bringing about health reform for CALD communities.

\section{Conclusion}

The themes identified in this paper bring new insights into the role of lay community navigators and their important, but demanding, place in any response to health access and equity for CALD communities, as outlined in the National Health Reform (2011) policy. Our study has demonstrated how a small group of community navigators are beginning to make a difference to health outcomes for their respective CALD communities in Logan, a disadvantaged region of Queensland. Even though the findings can only be considered in the context of the setting within which data were collected, the model is nevertheless transferable to similar areas where large numbers of CALD people live and where the demographic, socioeconomic and employment characteristics mirror that of Logan (Australian Bureau of Statistics 2001). Therefore, it may be appropriate to implement a similar model in these areas to improve health outcomes. The navigator model can function alongside other similar lay health worker models in Australia, such as chronic disease self-management peer leaders, but could also mirror the role of Aboriginal and Torres Strait Island health workers. There is evidence to support the use of lay health worker models in developing countries, such as Uganda, (Nabudere et al. 2011) to successfully promote maternal and child health as well as in developed countries, where such models are being used to address health professional shortages and to improve health care access (Liu et al. 2011).

\section{Conflicts of interest}

None declared.

\section{Acknowledgements}

The authors would like to thank Connecting Health in Communities, the Logan Beaudesert Health Coalition, Queensland Health and Griffith University for funding this project. We would also like to acknowledge the CEOs and staff of MultiLink Community Services Inc., Access Services Inc.; the Multicultural Coordinator of Logan-Beaudesert and the navigators who generously gave their time and shared their experiences. Thanks also to Felket Kahsay who conducted the learning circles and to Lesley Kelly who project managed the implementation phase of the model. 


\section{References}

Altpeter M, Earp JA, Bishop C (1999) Lay health advisor activity levels: definitions from the field. Health Education \& Behavior 26(4), 495-512. doi: $10.1177 / 109019819902600408$

Australian Bureau of Statistics (2001) National health survey: mental health, Australia. ABS catalogue no. 4811.0. (Australian Bureau of Statistics: Canberra)

Bishop C, Earp JA, Eng E, Lynch KS (2002) Implementing a natural helper lay health advisor program: lessons learned from unplanned events. Health Promotion Practice 3(2), 233-244. doi:10.1177/152483990200300218

Crotty M (1996) 'Phenomenology and nursing research.' (WB Saunders: Philadelphia, PA)

Department of Health and Ageing (2011) National Health Reform: improving primary health care for all Australians. (Australian Government: Canberra) Available at http://www.yourhealth.gov.au/internet/yourhealth/ publishing.nsf/content/nathealthref [Verified 20 June 2011]

Eng E, Parker E (2002) Natural helper models to enhance a community's health and competence. In 'Emerging theories in health promotion practice and research: strategies for improving public health'. (Eds RJ Diclemente, RA Crosby, MC Kegler) pp. 126-156. (Jossey-Bass: San Francisco, CA)

Federation of Ethnic Communities Councils of Australia (2009) FECCA submission on the national preventative health strategy discussion paper. Available at http://www.fecca.org.au/Submissions/2009/submissions 2009003.pdf [Verified 11 April 2011]

Gammonley D (2009) Psychological well-being and social support among elders employed as lay helpers. Journal of Gerontological Social Work 52, 64-80. doi:10.1080/01634370802561976

Giorgi A (Ed.) (1985) 'Phenomenology and psychological research.' (Duquesne University Press: Pittsburgh, PA)

Henderson S, Kendall E, See L (2011) Health and Social Care in the Community 19, 225-249. doi:10.1111/j.1365.2010.00972.x
Hibbert D, Catlin A, Chew-Graham C, Clarke P, Edwards S, Kovandzic M (2008) Access to high quality mental health services by hard-to-reach groups: a grey literature review. Available at http://www.liv.ac.uk/amp/ grey literature review.pdf [Verified 20 June 2011]

Lewin SA, Babigumira SM, Glenton C (2010) Lay health workers in primary and community health care: a systematic review. Available at http://www. cochrane.org/reviews/en/ab004015.html [Verified 13 April 2011]

Liu A, Sullivan S, Khan M, Sachs S, Singh P (2011) Community health workers in global health: scale and scalability. The Mount Sinai Journal of Medicine, New York 78(3), 419-435. doi:10.1002/msj.20260

Nabudere H, Asiimwe D, Mijumbi R (2011) Task shifting in maternal and child health care: an evidence brief for Uganda. International Journal of Technology Assessment in Health Care 27(2), 173-179. doi:10.1017/ S0266462311000055

Partridge MR, Caress AL, Brown C, Hennings J, Luker K, Woodcock A, Campbell M (2007) Can lay people deliver asthma self-management education as effectively as primary care based practice nurses? Thorax 63, 778-783. doi:10.1136/thx. 2007.084251

Reid K, Flowers P, Larkin M (2005) Exploring lived experience. Psychologist 18(1), 20-23.

Smith JA (2007) Hermeneutics, human sciences and health: linking theory and practice. International Journal of Qualitative Studies on Health and Well-being 2(1), 3-11. doi:10.1080/17482620601016120

Streubert-Speziali HJ, Carpenter DR (2006) 'Qualitative research in nursing: advancing the humanistic imperative. Edn 5.' (Lippincott Williams and Wilkinson: Philadelphia, PA)

Yu MY, Song L, Seetoo A, Cai C, Smith G, Oakley D (2007) Culturally competent training program: a key to training lay health advisors for promoting breast cancer screening. Health Education \& Behavior 34, 928-941. doi:10.1177/1090198107304577 
AUTHOR QUERIES

1. Please provide full postal address details for both affiliations A and B. 